\section{Cost-effectiveness of genetic studies in inherited heart diseases}

\author{
María Sabater-Molina, ${ }^{1}$ \\ Esperanza García-Molina, ${ }^{1}$ Isabel Tovar, ${ }^{1}$ \\ Francisco Ruiz-Espejo, ${ }^{1}$ \\ Juan Ramón Gimeno, ${ }^{2}$ Mariano Valdés ${ }^{3}$ \\ 'Servicio de Análisis Clínicos, Hospital \\ Universitario Virgen de la Arrixaca, \\ Murcia; ${ }^{2}$ Servicio de Cardiología, Hospital \\ Universitario Virgen de la Arrixaca, \\ Murcia; ${ }^{3}$ Departamento de Medicina \\ Interna, Universidad de Murcia, Murcia, \\ Spain
}

\section{Abstract}

There is a need to evidence the cost of genetic testing and know their profitability in order to establish criteria for priorizing access to genetic testing for these diseases. We determinated the cost per positive genotyping in 234 index cases with diagnosis of hypertrophic cardiomyopathy (HCM), arrhythmogenic right ventricular cardiomyopathy (ARVC), long-QT syndrome (LQTS), or Brugada syndrome (BS). The genetic tests of the most prevalent genes and the estimation of the costs of periodical screening in wildtype relatives (WT) were calculated. A total of 738 individuals (517 HCM, 76 ARVC, 71 LQTS and $74 \mathrm{BS)}$ from 234 probands were genotyped. The savings made by not having to perform the clinical testing of WT relatives exceeded the cost of genotyping for HCM families $€+220,710$, ARVC families $€+9405$ and LQTS families $€$ +8362 . The balance in BS was negative (€ $-25,112$ ). Our data suggests that individuals with conclusive clinical diagnostic of HCM should have a priority to access genetic testing. A positive overall benefit was also demonstrated in ARVC and LQTS.

\section{Introduction}

Inherited heart diseases are major causes of fatal arrhythmias and sudden death. ${ }^{1}$ Genetic diagnosis in cardiomyopathies is recommended by International Cardiac Societies., ${ }^{2,3}$ Although genetic information can help patient management and family study, its use is still limited by the expenses of DNA sequencing. In particular, genetic testing is useful for identifying both the relatives carrying the disease causing variant found in the families, who should be evaluated and followed-up, and the individuals without the mutation, who can be discharged.
Our aim was to analyse the costs of genetic testing (in probands and relatives) of the most prevalent genes involved in each of 4 inherited cardiac diseases and compare them with the costs of the clinical tests which could be saved during the follow-ups of relatives who do not carry the mutation. Cost-effectiveness was calculated for 2 cardiomyopathies [hypertrophic (HCM) and arrhythmogenic right ventricular (ARVC) cardiomyopathy] and 2 channelopathies [long-QT (LQTS) and Brugada syndrome (BS)]. The difference was calculated between the cost of genotyping and the savings made by not having to carry out clinical followups in the non-carriers [wildtype (WT)].

\section{Materials and Methods}

Two hundred thirty-four non-related probands [175 men, mean aged $( \pm \mathrm{SD})$ $40.4 \pm 18.1$ years ] with a diagnosis of HCM $(\mathrm{n}=115)$, ARVC $(\mathrm{n}=18)$, LQTS $(\mathrm{n}=17), \mathrm{BS}$ $(\mathrm{n}=84)$ were included in the study. Six of the 234 probands were from America, 2 from Asia and the rest of them from Europe. A percentage of seventy-nine of our population was Spanish origin. HCM patients with a family history of cardiomyopathy and/or sudden death in a young relative were prioritised. ARVC, LQTS, and BS were consecutively included regardless of the severity of the condition in the probands or the family history of complications. Complete double strand direct sequencing of all the coding exons and flanking intronic regions of the most prevalent genes was carried out in the 243 probands: HCM (MYH7 and MYBPC3), ARVD (PKP2, DSP, DSC2, DSG2 and $J U P)$, LQTS (KCNQ1, KCNH2, SCN5A, KCNE1, KCNE2, KCNJ2) and BS (SCN5A). DNA was extracted using standard protocols from peripheral blood samples. The yield of genetic testing was defined as the percentage of patients with positive genotyping. To assess the pathogenicity of unknown DNA variants we realized in silico study using three different softwares [Pmut, PolyPhen-2(v2.2.2r398), and MutationTaster] and in those cases in which was possible the cosegregation family study.

The cost per positive genotyping was calculated as the cost of sequencing the most prevalent genes in the probands divided by the number of patients with positive genotyping. DNA variants of uncertain significance were not included as positive-genetic results.

The expenses of sequencing were estimated from average prices in 2012 using data from two private companies operating in Spain (Table 1), ${ }^{4,5}$ Once the mutation was identified in the proband, a predictive test was recommended in relatives. The cost of the predictive test was estimated at $€ 250$ per individual.

The costs of basic clinical screening were
Correspondence: María Sabater Molina, Unidad de Cardiopatías Hereditarias, Hospital Universitario Virgen Arrixaca, Ctra Murcia-Cartagena s/n., El Palmar (30120), Murcia, Spain.

Tel. +34.968369441 - Fax: +34.968369418.

E-mail:mariasm@um.es

Key words: cost-effectiveness, genetic study, cardiomyopathies, channelopathies.

Contributions: MSM, EGM, laboratory work performing; JRG, clinical study performing. All authors participated in planning, conducting and reporting the work, reading and approving the final manuscript.

Funding: this study has been funded partly by a national grant of Sociedad Española de Cardiología and by the Cardiovascular Research Network (RECAVA) from the Health Institute Carlos III (C03/01, RD06/0014/0017, RD06/0014/0018).

Conflict of interests: the authors declare no potential conflict of interests.

Received for publication: 30 January 2013.

Revision received: 2 April 2013

Accepted for publication: 14 April 2013.

This work is licensed under a Creative Commons Attribution NonCommercial 3.0 License (CC BYNC 3.0).

(C) Copyright M. Sabater-Molina et al., 2013

Licensee PAGEPress, Italy

Cardiogenetics 2013; 3:e5

doi:10.4081/cardiogenetics.2013.e5

based on 2011 prices provided by the local Health Service. The following prices were quoted: an electrocardiogram (ECG) cost $€$ 51.4; a signal-averaged electrocardiography $€$ 77.3 ; a drug challenge test $€ 116$; an echocardiogram $€ 95.8$; an exercise test $€ 116$; and cardiac magnetic resonance $€ 119.7$. The cost of the first cardiac consultation was $€ 163.2$ and a follow-up consultation was priced at $€ 97.9$. The clinical work-up protocol varied according to the type of cardiomyopathy (Table 2$),{ }^{6}$ with standard 12-lead ECG and echocardiography being common in the first evaluation of all cardiomyopathies. Other tests were required for diagnosis in ARVC, LQTS and BS. Examinations performed in the follow-ups included an ECG, an echocardiogram and consultation for cardiomyopathies, with only an ECG and consultation for channelopathies.

The estimated cost of periodical screening in WT relatives was calculated by taking into account the expenses of basic routine tests throughout their lifetime from 10-60 years of age, frequency was based on European Society of Cardiology recommendations (Table 2). ${ }^{6}$

The cost of other clinical tests not included in the protocol (Table 2$)^{6}$ in WT relatives was 
not included in the analysis. The expenses of clinical examination in probands or in carriers were not included in the analysis. The cost of genetic testing in relatives found to be carriers was also excluded from the analysis.

All patients provided signed informed consent and received genetic counselling from expert staff. Protocol was approved by the medical centre ethics committee. All individuals were evaluated in a specialist inherited cardiac disease unit.

It was decided not to include dilated cardiomyopathy in the study, due to the large number of genes required and the low expected diagnostic yield, which suggested very negative cost-effectiveness a priori.

\section{Results}

Disease causing mutations were identified in the following probands: 92 out of $115(80 \%)$ with HCM, leading to a cost per positive genotyping of $€ 1795$; 14 out of 18 (78\%) with ARVC, with a cost per positive genotyping of $€ 2898$; 11 out of $17(65 \%)$ with LQTS, with a cost per positive genotyping of $€ 2715$; and in 16 out of 84 (19\%) with BS, with a cost per positive genotyping of $€ 3696$. The mutations found in the different genes were: MYBPC3 (12 missense mutations: G148R, A216T+E258K, E441K, R502Q, E542Q, V771M, M844R, N948T, R1022P, E1179K, G1248R, 6 type non-missense: A107fsX116, K505del, W818-R820delWMR, E838X, G853RfsX31， R891AfsX160 and 2 intronic mutations: IVS20-2A>G, IVS23+ 1G>A); MYH7 (8 missense mutations: D309G, V606M，D928N，G1057D，E1348Q，E1356Q, T1377M, R1382Q); PKP2 (2 missense mutations: S140F, A418D and 2 non-missense: R79X, V837fsX930); DSP (3 non-missense mutations: R425X, A1074SfsX1087, Q1925X); JUP (one missense mutation: T19I); KCNQ1 (3 missense mutation: P197L, R533W, N586S and 1 intronic mutation: IVS6+1G>C); KCNH2 (3 missense mutations: T13N, H562R, C566G); SCN5A (6 missense mutations: R27H, E746K, E901K, V1338I, N1443S, G1743R and one nonmissense: E1151X) and KCNJ2 (1 missense mutation: N410S). Three probands (3.3\%) with diagnostic of HCM presented double mutation in the MYBPC3 gene (A216T and E258K). A total of 738 individuals from 234 index subjects (3.1 per family) were genotyped. The following proportion of relatives were tested: 224 WT out of $517 \mathrm{HCM}$ relatives (22\%); 40 WT out of 76 ARVC relatives (53\%); 36 WT out of 71 LQTS relatives (51\%); and 41 WT out of 74 (55\%) BS relatives (Figure 1). The savings made from not having to carry out the periodical clinical screening of 224 WT relatives from HCM families were higher than the costs of genetic testing which included the genetic tests of probands and the predictive genetic tests of WT relatives ( $€ 441,850$ $221,140=€+220,710$ ) (Figure 2). Similarly, the savings made by not having to perform the clinical testing of 40 WT relatives from ARVC families exceeded the cost of genotyping $(€ 59,977-50,572=€+9405)$. There was also a positive cost-effectiveness in LQTS families: the savings made by not having to carry out the clinical testing of 36 WT relatives from LQTS families was greater than the cost of the genetic tests (€47,241-€38,869=€+8362). However, the expenses of genotyping in BS families exceeded those of the clinical testing of WT relatives, resulting in a negative effect (€42,274-67,386=€-25,112).

\section{Discussion}

Our results support the recommendation of consensus documents ${ }^{3}$ that genetic testing should be performed on the majority of HCM patients as this can be of help for both identifying carriers and ruling out non-carriers with implications for genetic counselling. Furthermore, this decision is cost-effective given that the estimated expenses of the genetic testing are outweighed by the savings made from not having to perform from repetitive clinical examinations in WT relatives who could be discharged.

The genotyping yield is substantially high in ARVC and LQTS suggesting that patients with these two conditions should be studied genetically too, although there is less cost-effectiveness compared to HCM. The proportion of positive testing in BS is still low which leads to a small number of WT relatives being discharged and a negative cost-effectiveness.

In our study, we decided to limit the analysis of MYBPC3 and MHY7 for HCM patients as the pick-up rate in the first series of cases demonstrated a high diagnostic yield, probably due to the presence of founder mutations in the area, and a selection bias towards the more severe cases. The inclusion of 5 sarcomeric genes (MYBPC3, MYH7, TNNI3, TNNT2, TPM1) as recommended by other authors, ${ }^{6}$ instead of 2 , would have resulted in a lower, but probably still positive cost-effectiveness as a result of genetic testing in HCM families as it has been previously reported by Wordsworth et al. ${ }^{2}$

Genetic testing helps to reduce the cost of the screening of selected cardiomyopathies and channelopathies. Conversely, the genetic information does not change throughout the relative's lifetime, and the identification of the disease-causing variant may occur before the onset of symptoms.

The analysis of the clinical relevance of genetic testing is beyond the scope of this study. The clinical benefit of genetics in BS and in all inherited cardiac conditions should be considered individually. ${ }^{8}$ The yield of genotyping and thus the cost-effectiveness in BS increases in selected patients who have a coexisting atrioventricular block. ${ }^{9}$

The analysis of the cost-effectiveness of

Table 1. Genetic protocol for affected probands and average costs.

\begin{tabular}{ccccc} 
& HCM & ARVC & LQTS & BS \\
Genes & MYBPC3 & PKP2 & KCNQ1, KCNH2, SCN5A, KCNE1, & SCN5A \\
& $M Y H 7$ & DSP, DSC2, DSG2, PKG & KCNE2, KCNJ2 & \\
Cost ( $)$ & 1436 & 2254 & 1757 & 704 \\
\hline
\end{tabular}

HCM, hypertrophic cardiomyophathy; ARVC, arrhythmogenic right ventricular cardiomyopathy; LQTS, long-QT syndrome; BS, Brugada syndrome.

Table 2. Clinical work-up protocol for non-affected first-degree relatives of a patient with cardiomyopathy based on recommendations from the European Society of Cardiology consensus document in 2010.

$\begin{array}{ccccc} & \text { HCM } & \text { ARVC } & \text { LOTS } & \text { BS } \\ 1^{\text {st }} \text { cardiac examination } & \text { ECG } & \text { ECG } & \text { ECG } & \text { ECG } \\ & \text { Echocardiogram } & \text { SAECG } & \text { Echocardiogram } & \text { Echocardiography } \\ & & \begin{array}{c}\text { Echocardiogram } \\ \text { CMR }\end{array} & \text { Exercise test } & \text { Drug challenge test }\end{array}$

\begin{tabular}{lccc} 
Follow-up examination & $\begin{array}{c}\text { ECG } \\
\text { Echocardiogram }\end{array}$ & $\begin{array}{c}\text { ECG } \\
\text { Echocardiogram }\end{array}$ & ECG \\
\hline Repeated examinations & Annually in 10 to 20 years old \\
& Every 3 years in 20 to 60 years old \\
\hline HCM, hypertrophic cardiomyophathy; ARVC, arrhythmogenic right ventricular cardiomyopathy; LQTS, long-QT syndrome; BS, Brugada syndrome; \\
ECG, electrocardiogram; SAECG, signal-averaged ECG; CMR, cardiac magnetic resonance.
\end{tabular}
ECG, electrocardiogram; SAECG, signal-averaged ECG; CMR, cardiac magnetic resonance. 
genetic testing might vary substantially from country to country, and will probably change with the introduction of new sequencing technologies. Despite ARVC cases were consecutively enrolled referral bias towards the most severe cases of this series could explain the high pick-up rate.

\section{Conclusions}

Individuals diagnosed with conclusive clinical HCM should have priority access to genetic testing given that the benefits of savings made from not having to perform periodical tests on wildtype relatives who can be discharged, clearly exceed the expenses of genetic testing. A positive overall benefit has also been demonstrated in ARVC and LQTS.

\section{References}

1. Gimeno JR, Oliva MJ, Lacunza J, et al. Characteristics of sudden death in inherited cardiac diseases. Rev Esp Cardiol 2010;63:268-76.

2. Wordsworth S, Leal J, Blair E, et al. DNA testing for hypertrophic cardiomyopathy: a cost-effectiveness model. Eur Heart J 2010;31:926-35.

3. Ackerman MJ, Priori SG, Willems S, et al. HRS/EHRA expert consensus statement on the state of genetic testing for the channelopathies and cardiomyopathies. Heart Rhythm 2011;8:1308-39.

4. Health In Code (HIC). Cost-benefit analysis. Coruña: Health In Code; 2011. Available from: http://www.healthincode. com/images/stories/pdf/hic006_cost-benefit_analysis.pdf

5. Suddincode. Conocer la predisposición genética a la muerte súbita cardíaca, hoy es posible. Barcelona: Ferrer In Code; 2013. Available from: http://www.ferrerincode.com/es_ES/index.asp

6. Richard P, Charron P, Carrier L, et al. EUROGENE Heart Failure Project. Hypertrophic cardiomyopathy: distribution of disease genes, spectrum of mutations, and implications for a molecular diagnosis strategy. Circulation 2003;107:2227-32.

7. Oliva MJ, Ruiz-Espejo F, Monserrat L, et al. Insights into genotypeephenotype correlation in hypertrophic cardiomyopathy.

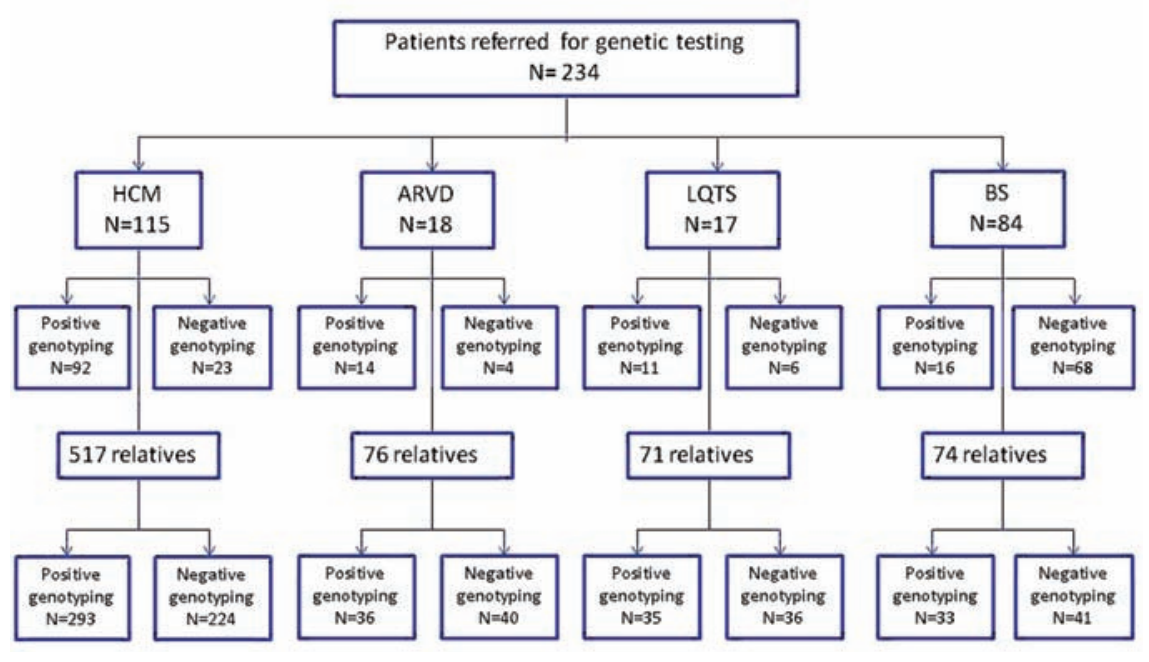

Figure 1. Flowchart describing the study population and the genotyping yields in the different subgroups of patients screened for mutations on genes associated with hypertrophic cardiomyophathy (HCM), arrhythmogenic right ventricular cardiomyopathy (ARVC), long-QT syndrome (LQTS) and Brugada syndrome (BS).

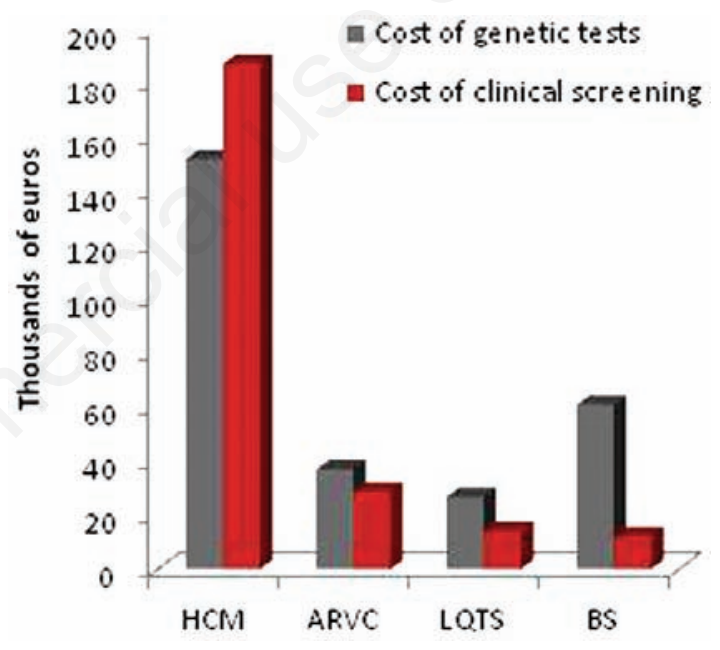

Figure 2. Cost in thousands of euros of the clinical screening and genetic testing in genes associated with hypertrophic cardiomyophathy (HCM), arrhythmogenic right ventricular cardiomyopathy (ARVC), long-QT syndrome (LQTS) and Brugada syndrome (BS).

Findings from 18 Spanish families with a single mutation in MYBPC3. Heart 2010; 96:1980-4.

8. Charron P, Arad M, Arbustini E, et al. Genetic counselling and testing in cardiomyopathies: a position statement of the European Society of Cardiology Working
Group on Myocardial and Pericardial Diseases. Eur Heart J 2010;31:2715-26.

9. Bai R, Napolitano C, Bloise R, et al. Yield of genetic screening in inherited cardiac channelopathies: how to prioritize access to genetic testing. Circ Arrhythm Electrophysiol 2009;2:6-15. 